\title{
Illumination of The Relationship between Ureter and Intervertebral Disc by Computed Tomography
}

\author{
Safiye Sanem DERELI BULUT ${ }^{1}$, Zakir SAKCI ${ }^{1}$, Sait NADERI ${ }^{2}$ \\ ${ }^{1}$ Health Sciences University, Istanbul Umraniye Training and Research Hospital, Department of Radiology, Istanbul, Turkey \\ ${ }^{2}$ Health Sciences University, Istanbul Umraniye Training and Research Hospital, Department of Neurosurgery, Istanbul, Turkey
}

Corresponding author: Safiye Sanem DERELI BULUT ssanembulut@gmail.com

\section{ABSTRACT}

AIM: To show the relationship of lumbar intervertebral discs with the ureters by using computerized tomography (CT).

MATERIAL and METHODS: Eighty patients (male/female: 42/38) with a mean age of 45 years were included in this retrospective study. An imaginary line passing through the tangential to the posterior cortex of the vertebral body was drawn at the intervertebral disc level (line a). Perpendicular to this line were two lines (line b) drawn from the ureters. The lines of the cortex, where "line a" was cut (point c), were also drawn from the contralateral ureter (line $d$ ). The distances between "lines $b$ and d" were measured. Another line was drawn from both "c" points to the ipsilateral ureters (line $x$ ). The angle between "lines a and x" was measured (angle $x$ ).

RESULTS: The right kidney hilus was inferior to the left kidney hilus. A significant negative correlation was observed between line band lumbar level $(r=-0.95)$. The average length of "line b" at the lower lumber levels was $2.1 \mathrm{~cm}$. Moreover, a positive correlation was found between "angle $x$ " and lumbar level $(p<0.05)$. The "angle $x$ " increased from $38^{\circ}$ to $80^{\circ}$ with the decrease in lumbar levels. In addition, the "angle $x$ " was significantly higher in male patients $(p<0.05)$ than in female patients. No significant correlation was found between "line d" and lumbar level.

CONCLUSION: CT is an illumination modality that could elucidate the relationship between intervertebral disc and ureter preoperatively and could visualize the retroperitoneum.

KEYWORDS: Ureter, Ureter injury, Computed tomography, Spinal surgery

\section{INTRODUCTION}

$\mathrm{T}$ The ureters are rarely exposed to trauma due to their anatomical location. When they occur, ureteral injuries are classified in the order of decreasing frequency as follows: iatrogenic injuries, blunt injuries, and penetrating injuries $(6,7,12)$, whereas ureteral injuries have been reported after a microdiscectomy and after a posterolateral lumbar approach that was employed to address disc herniations (8).

A ureteral injury has no specific clinical signs and therefore its symptoms occur late. Only one-third of ureteral injuries are noticeable during surgery $(7,12)$.

In increasing frequency, ureteral injuries occur in the distal, middle, and proximal regions of the ureter $(1,5)$. To avoid ureteral injuries, surgeons should carefully examine the ureteral anatomy, supply network, and adjacencies. In females, the ureters are located adjacent to the reproductive organs and thus are threatened during liberation (6). In case of a suspected injury, the radiologic diagnostic tools that may be used include ultrasonography (USG), computed tomography (CT), or intravenous pyelography $(5,6)$.

When a ureteral injury is suspected during the postoperative period, diagnosis can be made using various radiological modalities, starting with USG to detect urinomas, abscess formations, and hydronephrosis. The other imaging modalities that may be employed to determine the degree of injury include contrast-enhanced CT and CT urography. As regards treatment, the approach employed varies depending on the localization of a trauma $(1,5,9)$. 
The ureter is normally located lateral to the aorta on the left and lateral to the inferior vena cava (IVC) on the right. At the L4-L5 level, it is located between the anterolateral aspects of the vertebral body (VB) and the psoas muscle (PM). It crosses the common iliac artery and vein ventrally, and it is located medial to these vessels at the lumbosacral junction. Consequently, ureteral injuries are sometimes associated with combined arterial or venous injuries and vice versa $(2,14)$.

This study aimed to show the relationship of the vertebral pedicle and intervertebral discs with the ureters by using CT and to show that CT is an illumination modality for surgeons during the preoperative period.

\section{MATERIAL and METHODS}

\section{Patients}

This research is a single-center, cross-sectional, retrospective study. A total of 80 consecutive patients who underwent abdominal CT scan for renal stones from May 1, 2017 to January 1, 2018, were included in this study.

The exclusion criteria were as follows: patients with hydronephrosis or an abnormality in the urinary system (e.g., ectopia, duplication, aplasia, and hypoplasia), renal failure, renal cystic diseases, intra-abdominal malignancy, history of intra-abdominal operation, and lumbar scoliosis.

Patients who underwent abdominal CT due to condition other than those listed above and who had two normal kidneys were included. An approval from the concerned ethics committee was sought (No. 54132726-000-17574), and informed written consent was obtained from all of the included patients.

\section{CT Technique}

CT scans were performed in a multi-detector scanner (128-slice GE Optima CT660 CT scanner; GE Healthcare). The CT scans were obtained in the supine position during which the the patients were instructed to hold their breath to minimize motion artifacts. The CT protocols were as follows: tube voltage, $120 \mathrm{kVp}$; automatic tube current modulation, 30-70 mAs; pitch, 0.99-1.22 mm; matrix, $512 \times 512$; slice thickness, $10 \mathrm{~mm}$; and field of view, $380 \mathrm{~mm}$ (distance between $1 \mathrm{~cm}$ superior of the diaphragm to the femoral trochanter majus) (Table I). All images were subsequently reconstructed with a slice thickness of $2.5 \mathrm{~mm}$.

\section{Image Analysis}

Image datasets were exported to a Picture Archiving and Communication System (PACS) workstation for analysis (Centricity ${ }^{\circledR}$ PACS; GE Healthcare, Milwaukee, WI, USA).

The images were evaluated by a radiologist with 10 years of experience in abdominal radiology.

The patients were divided into two groups: males (Group 1) and females (Group 2). Each group was further divided into two subgroups: patients aged below 55 years (Group 1A and Group 2A) and those aged above 55 years (Group 1B and Group 2B).
The reference section at each intervertebral disc level was obtained by reconstructing the images parallel to the end plates of the vertebras. While the end plateaus at the L2-L3 level were oriented more transversely, they were more oblique at the L5-S1 level. This reference cross section was planned by taking the opening into consideration (Figure 1).

An imaginary line passing through the tangential to the posterior cortex of the corpus at the intervertebral disc level was drawn (line a). Perpendicular to this line were two lines drawn from the ureters (line $b$ ). Also, the lines of the cortex, where linea was cut (point c), were drawn from the contralateral ureter (line $d$ ). The distances between lines $b$ and $\mathrm{d}$ were measured. Another line was drawn from both $\mathrm{c}$ points to the ipsilateral ureters (line $x$ ). Moreover, the angle between lines $a$ and $x$ was measured (angle $x$ ) (Figures 2A-C).

The ureters originating from both renal pelvises were marked with a circular region of interest (ROI) for each level of lumbar intervertebral discs. Both ureters were followed in the craniocaudal direction through all sections and marked with circular ROls. All measurements were repeated separately for each lumbar intervertebral disc level. All measurements were made separately for the right and left sides.

\section{Statistical Analysis}

All statistical analyses were performed using the Statistical Package for the Social Sciences (SPSS) software (version 22.0; SPSS Inc., Chicago, IL, USA).

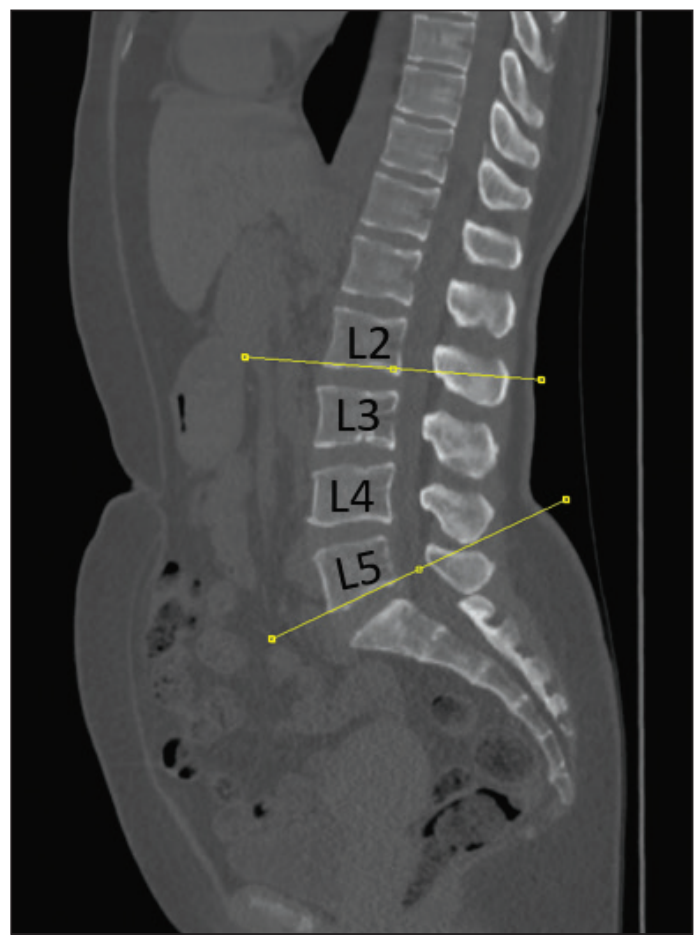

Figure 1: Planning for the sections tangent to the lower end plateau at the L2-L3 intervertebral disc (upper yellow line) and L5$\mathrm{S} 1$ intervertebral disc (lower yellow line) levels. The cross section to be used in measuring the ureteric distance was oriented parallel to the lower end of the vertebral plateau. The L5-S1 level is more inclined than the upper levels. 

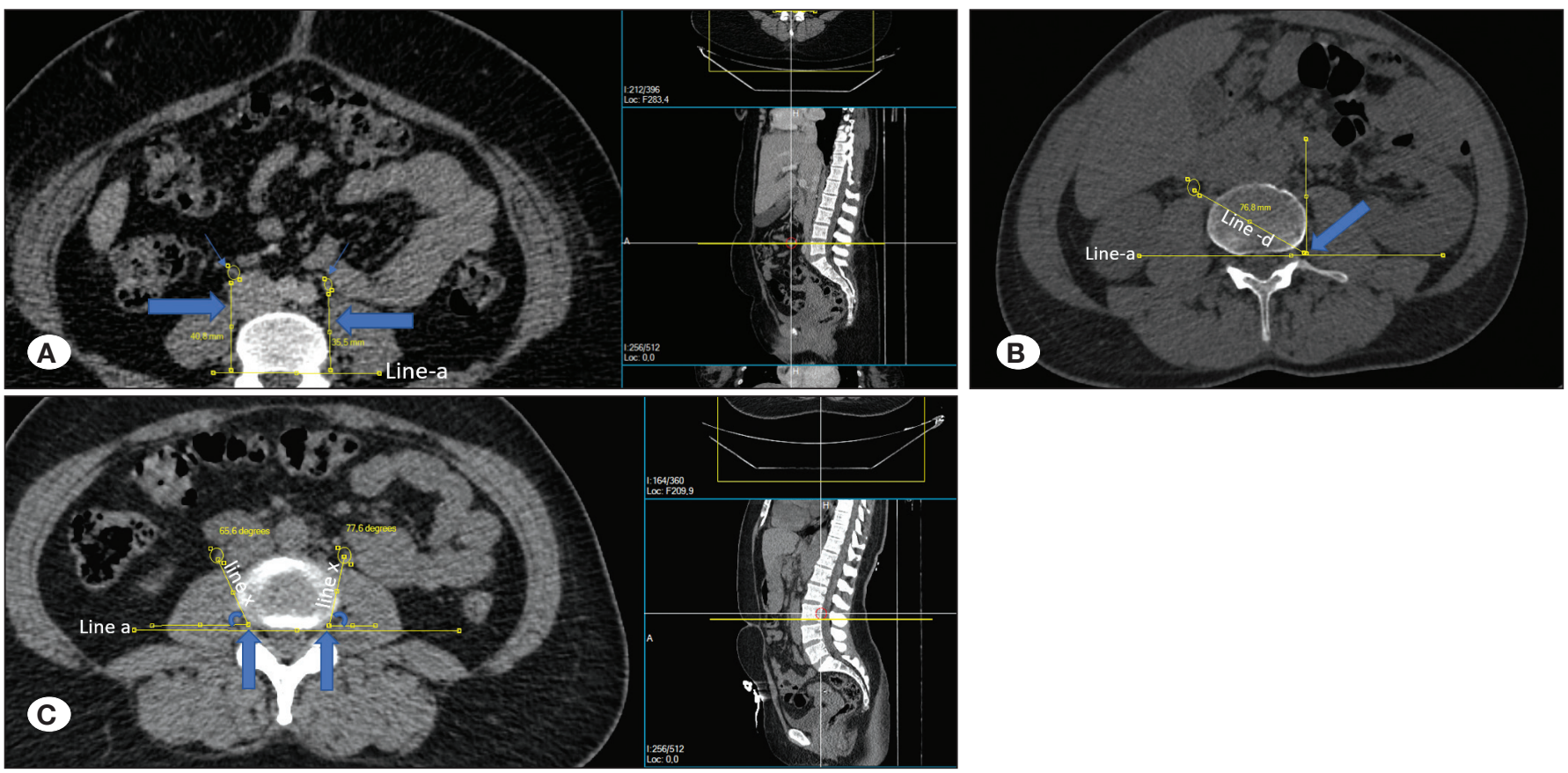

Figure 2: A) Illustrative case of a 37-year-old male patient. In the section passing through the L4-L5 intervertebral disc level, both ureters are marked with yellow circular ROIs (thin blue arrows). The vertebra is followed by line a tangent to the corpus posterior cortex. There are $b$ lines from both ureters extending perpendicular to this line (thick blue arrows). The line on the right measured $40.8 \mathrm{~mm}$ and that on the left measured $35.5 \mathrm{~mm}$. The lines in the cross section passing through the L4-L5 intervertebral disc level are seen in the image. B) Illustrative case of a 41-year-old female patient. The section passing through the L3-L4 intervertebral disc could be seen. Point c is where line a tangent to the vertebra corpus posterior cortex intersects the lateral cortex (thick blue arrow). The line extending from the contralateral ureter to the point $\mathrm{c}$ is the line $\mathrm{d}$. In this patient, line $\mathrm{d}$ measured $76.8 \mathrm{~mm}$ on the right ureter. The procedure was repeated for the left ureter. C) Illustrative case of a 53-year-old male patient. In the axial plan, the section passes through the L3-L4 level. Both ureters were marked with small circular yellow ROls. There are lines (line $\mathrm{x}$ ) extending from both ureters going to the ipsilateral $\mathrm{c}$ points (long thick blue arrows). The angle between line $\mathrm{x}$ and line $\mathrm{a}$ is the anglex (marked by curved blue arrows). In this patient, angle $\mathrm{x}$ was $65.6^{\circ}$ for the right ureter and $77.6^{\circ}$ for the left ureter.

The distribution of the outcome categories was assessed using the Shapiro-Wilk test. Data are presented as mean \pm standard deviation based on the normality of data. Categorical variables are presented as counts and percentages.

Statistical significance is indicated by $p<0.05$.

\section{RESULTS}

Among the 80 included patients, 42 were male (Group 1A: 24; Group 1B:18) and 38 were females (Group 2A: 21; Group 2B: $17)$ with a mean age of 45 and 48 years, respectively.

In all groups, the right kidney was lower than the left kidney. With the vertebral body (VB) as reference, the left renal pelvis is generally located at the midlevel of the VB, whereas the right renal pelvis is located at the lower end plateau of the VB.

In some of our patients, no ureter matching was observed in the L1-L2 intervertebral disc level. Moreover, the ureteropelvic junction was inferior to this level. Measurements were therefore made starting at the L2-L3 intervertebral disc level (Figure 3).

In Group 1, the average distances of line $b$ were $45 \mathrm{~mm}$ to $23 \mathrm{~mm}$ for Group $1 \mathrm{~A}$ and $42 \mathrm{~mm}$ to $25 \mathrm{~mm}$ for Group 1B. The distances of line $b$ were negatively correlated with lumbar level $(r=-0.78, p<0.05)$.
In Group 2, the average distances of line $b$ were $46 \mathrm{~mm}$ to $22 \mathrm{~mm}$ in Group 2A and $38 \mathrm{~mm}$ to $25 \mathrm{~cm}$ for Group 2B. A negative correlation was also found between the lumbar level and the line $b$ distances $(r=-0.63, p<0.05)$.

Similarly, a significant negative correlation was found between line $b$ and lumbar level $(r=0.54, p<0.05)$. A significant negative correlation was also found between line $b$ distances and angle $x$ on the left side $(r=-0.48, p<0.05)$.

By contrast, a significant positive correlation was found between lines $b$ and $d(r=0.57 ; p<0.05)$.

Line $d$ and angle $x$ on the right side were also significantly negatively correlated $(r=-0.56, p<0.05)$.

On the left side, a positive correlation was observed between angle $x$ and lumbar levels $(r=0.48, p<0.05)$. The angle $x$ increased from $38^{\circ}$ to $85^{\circ}$ toward the lower lumbar levels in both groups. No significant correlation was found between lumbar levels and angle $x$ on the right side $(p>0.05)$.

Furthermore, no significant correlation was found between line $d$ and lumbar level ( $p>0.05)$. In all groups, line $d$ increased with age $(r=0.61 p<0.05)$.

The results are summarized in Tables II and III. 
Table I: CT Protocol of Abdomen/Pelvis for Renal Stone

\begin{tabular}{ll}
\hline Topogram Direction & Craniocaudal \\
\hline Respiratory Phase I & Inspiration \\
\hline Scan Start / End Locations & $1 \mathrm{~cm}$ superior to diaphragm to the iliac crest (scan through entire kidneys) \\
\hline FOV (Field of view) & $38 \mathrm{~cm}$ (decrease appropriately) \\
\hline IV(intra venous) Contrast agent administration & non \\
\hline Scan Delay & non \\
\hline
\end{tabular}

Table II: Average Distances of b- Line and d- Line with SD Values According to Lumbar Levels and Groups

\begin{tabular}{ccccccccc}
\hline & \multicolumn{3}{c}{ b- line distances (mm) } & \multicolumn{3}{c}{ d -line distances (mm) } \\
\hline & Group 1A & Group 1B & Group 2A & Group 2B & Group 1A & Group 1B & Group 2A & Group 2B \\
\hline L2-L3 & $75 \pm 7$ & $59 \pm 6$ & $61 \pm 16$ & $40 \pm 13$ & $67 \pm 2$ & $66 \pm 7$ & $65 \pm 6$ & $69 \pm 4$ \\
\hline L3-L4 & $51 \pm 5$ & $33 \pm 7$ & $46 \pm 6$ & $52 \pm 11$ & $54 \pm 6$ & $56 \pm 2$ & $58 \pm 7$ & $67 \pm 3$ \\
\hline L4-L5 & $43 \pm 12$ & $32 \pm 5$ & $41 \pm 13$ & $38 \pm 5$ & $59 \pm 11$ & $64 \pm 3$ & $64 \pm 8$ & $68 \pm 5$ \\
\hline L5-S1 & $32 \pm 3$ & $27 \pm 8$ & $35 \pm 7$ & $24 \pm 3$ & $66 \pm 5$ & $68 \pm 3$ & $65 \pm 4$ & $66 \pm 8$ \\
\hline
\end{tabular}

A total of 80 patients are grouped by gender (group 1: male patients, group 2: female patients). In addition, patients in both groups were divided into two groups, those under the age of 55 and those above (group $1 A$ and $B$, and group $2 A$ and $B$ ). According to table, the decrease in the $b$-line is noteworthy as the level of lumbar and age increases. It is especially evident in female patients. The d-line increases with age.

Table III: X-Angle Mean Values and SD Values Measured from the Left and Right Sides in Lumbar Levels

\begin{tabular}{lcccccccc}
\hline & \multicolumn{3}{c}{ X- angle in the left side (degrees) } & \multicolumn{3}{c}{ X- angle in the right side (degrees) } \\
\hline & Group 1A & Group 1B & Group 2A & Group 2B & Group 1A & Group 1B & Group 2A & Group 2B \\
\hline L2-L3 & $70 \pm 6$ & $71 \pm 8$ & $68 \pm 11$ & $53 \pm 9$ & $75 \pm 6$ & $77 \pm 9$ & $65 \pm 11$ & $69 \pm 3$ \\
\hline L3-L4 & $68 \pm 11$ & $70 \pm 6$ & $71 \pm 5$ & $56 \pm 8$ & $68 \pm 3$ & $76 \pm 7$ & $60 \pm 7$ & $62 \pm 5$ \\
\hline L4-L5 & $78 \pm 5$ & $66 \pm 13$ & $67 \pm 6$ & $65 \pm 9$ & $67 \pm 4$ & $74 \pm 11$ & $63 \pm 9$ & $60 \pm 7$ \\
\hline L5-S1 & $72 \pm 9$ & $69 \pm 9$ & $68 \pm 7$ & $58 \pm 12$ & $65 \pm 4$ & $60 \pm 9$ & $64 \pm 8$ & $52 \pm 3$ \\
\hline
\end{tabular}

According to the table, the angle $x$ value on the left increases with increasing lumbar level. As the age increases, the $X$ angle decreases for both groups.
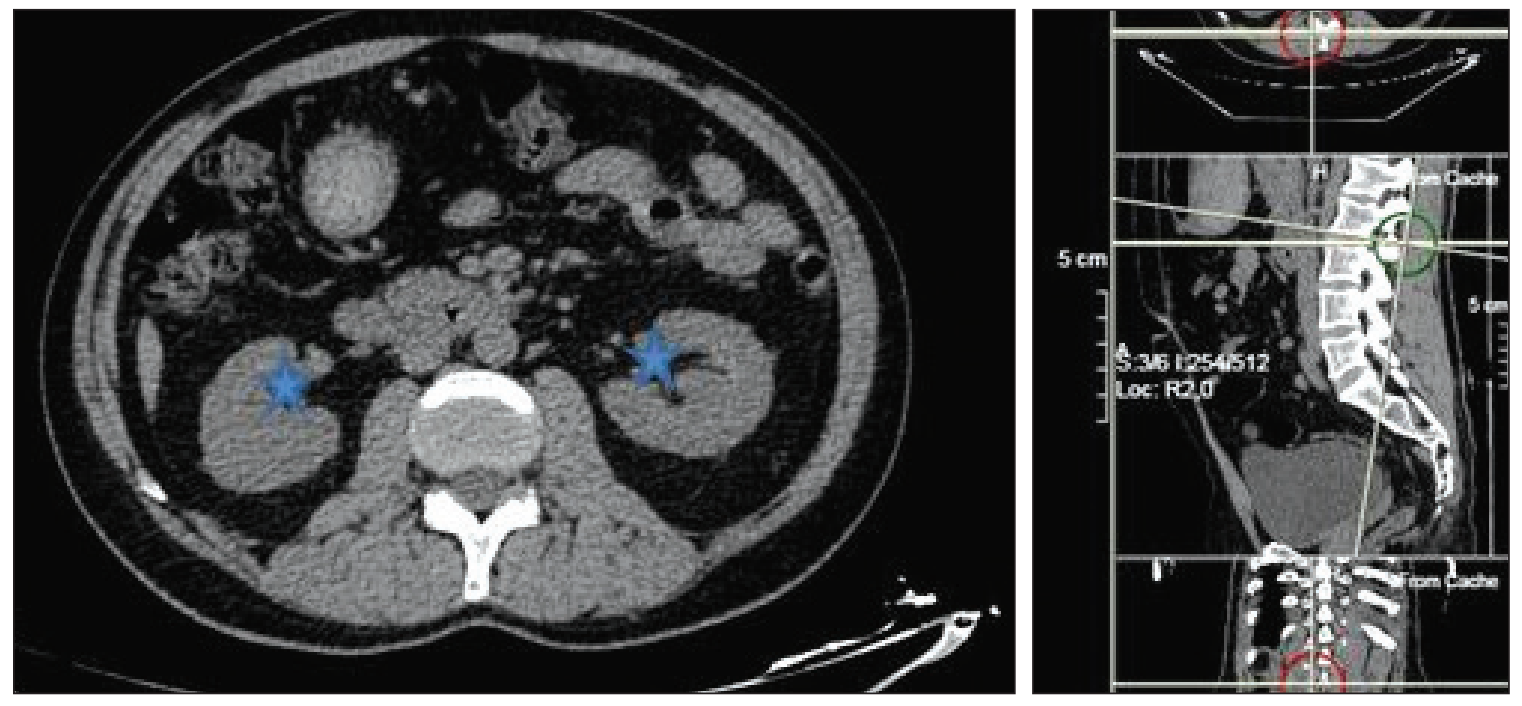

Figure 3: Renal hiluses (small blue stars) could be observed on axialplane CT section obtained from the L2-L3 level of the intervertebral disc. It is noteworthy that the ureters could not be observed in this section. 


\section{DISCUSSION}

Some small series in the literature have investigated ureteral injuries caused by anterior lumbar procedures. Given the retroperitoneal location of the ureter and the importance of the vascular structures adjacent to it, it is extremely important to protect the ureters, especially when anterior and posterolateral spine surgical approaches are employed (1).

We aimed to show the relationship of the vertebral pedicleintervertebral disc with the ureters by using CT. In addition, we aimed to show that CT could be an illumination modality for surgeons in the preoperative period.

Our results showed that line $b$ was higher in the upper lumbar levels and in Group 1 than in the lower lumbar levels and in Group 2. This result may be due to the PM volume being more visual, especially in young men. We speculate that the increased muscle volume, especially in young men, contributes to the distance of the ureter located in front of the psoas from the vertebral cortex. We also observed that the decreasing retroperitoneal fat tissue on the right side caused the ureter to approach the back, decreasing the value of the angle $x$. Moreover, the ureter was found close to the VB in weak patients with low retroperitoneal adipose tissue. This finding is supported by other studies $(1,5,13)$.

For the same reason, the value of angle $x$ is also significantly higher in young male patients.

Ureteral anatomy should be well examined by all surgeons from all disciplines who are interested in this region and in the retroperitoneal space $(1,6)$.

The number of anterior lumbar spinal reconstructions for degenerative disc diseases has significantly increased in the last two decades. The evolution of spine technology has changed how an occasional anterior procedure is performed wherein a large flank incision is made in spinal fusion surgery involving a direct anterior extraperitoneal exposure. Considering the proximity of the lumbar spine to the aorta, inferior vena cava, and iliac vessels, vascular surgeons frequently perform anterior exposures (15). The rapid increase in anterior spine surgery has created a demand for access surgeons who are familiar with retroperitoneal procedures and who are skilled at mobilizing vessels $(4,15)$.

The trans-psoas approach provides an undisturbed plane that allows access to the lateral disc space and is therefore a preferable approach to prevent ureter damage $(4,8,10)$.

One approach that could prevent ureter injuries involves the use of prophylactic ureteral stents and catheters in the preoperative period. Some surgeons may prefer this technique to detect injury during surgery. However, there is no consensus as regards the use of this technique. Some surgeons do not prefer this approach because of stent-related complications. (3).

According to the European Association of Urology guideline, preoperative prophylactic stent application is recommended in selected cases (based on risk factors and on surgeons' experience) to prevent ureter trauma. However, the guidelines of the American Society of Colon and Rectal Surgeons on performing surgeries for diverticulitis state that "ureteral stents are used at the discretion of the surgeon" $(3,5)$.

In a comprehensive study, it was mentioned that the application of prosthetic ureteral stents in patients undergoing colorectal surgery increases the treatment costs. In addition, many complications, such as hematoma, infection, and ureter laceration, may occur after a stent application (3). Thus, a noninvasive, easily accessible, reliable, and less costly method is highly desirable.

Determining the course of ureters in the preoperative period for each patient is important. In addition, safe areas in both retroperitoneal spaces in terms of the instrumentation to be used must be identified, especially before a spinal surgery. The ureter is normally located lateral to the aorta on the left and lateral to the IVC on the right. It is located between the anterolateral of the VB and PM at the L4-L5 level. At the lumbosacral junction level, it crosses the common iliac artery and vein ventrally. Consequently, ureteral injuries are sometimes accompanied by vascular injuries (2).

Ureteral injuries arise when the prevertebral or anterior longitudinal ligament is perforated by instruments used to cleara disc space. The most common location of injuries is the distal third of the ureter at the L4-L5 level. At this level, the ureter is closest to VB $(3,11)$.

The ureter lies within the retroperitoneal fat layer and is normally mobile. In lean patients, it can be located closer to the VB. In such patients, the probability of damage to the ureters located between the VB and the PM increases. Tilting a patient while in the prone position during surgery brings the ureter closer to the VB and to the intervertebral disc plane. Therefore, the risk of ureter damage may increase. Typically, an injured ureter is contralateral to the side where diskectomy is performed because of the tangential passage of the instrument during the procedure (2).

When necessary, patients may be placed in the prone position during operation, and the course of the ureter can be monitored in this position.

Our study has some limitations. First, our patient population is small. Second, no pathologies, such as spinal tumors or herniated discs, were found in our patient group. For this reason, we cannot describe our experiences before and after surgery.

In addition, a CT scan was performed without the administration of an intravenous contrast agent. However, the fact that the part of the examined ureter is found in front of the PM and is completely surrounded by retroperitoneal fatty tissue has made the tracing easier. In addition, thanks to multiformat reconstruction, the entire course of both ureters were easily traced. However, in some cases, a CT scan should be performed in the pyelogram phase when the location of the ureter needs to be defined clearly.

\section{CONCLUSION}

Defining the course of the ureter in the retroperitoneum is extremely important for each patient, especially when 
determining the proximity of the ureter to a spinal level that is to be operated. In this regard, CT can guide surgeons during the preoperative period.

\section{REFERENCES}

1. Burks FN, Santucci RA: Management of iatrogenic ureteral injury. Ther Adv Urol 6(3):115-124, 2014

2. Cho $\mathrm{K}, \mathrm{Im} \mathrm{SH}$, Hong SK: Ureteral injury after inadvertent violation of the intertransverse space during posterior lumbar diskectomy: A case report. Surg Neurol 69(2):135-137, 2008

3. Croghan SM, Zaborowski A, Mohan HM, Mulvin D, Mcguire BB, Murphy M, Galvin DJ, Lennon G, Quinlan D, Winter DC: The sentinel stent? A systematic review of the role of prophylactic ureteric stenting prior to colorectal resections. Int J Colorectal Dis 34(7):1161-1178, 2019

4. Dhar A, Pawar S, Prasad A, Ramani PS: Approaches to paraspinal tumours - a technical note. Neurological Research 39(4):315-322, 2017

5. Durmaz $\mathrm{H}$ : External-internal ureteral catheterization technique in treatment of ureteral injuries. Turk J Med Sci 49(6):11321137,2019

6. Ergani B, Turk H, Isoglu CS, Karabicak M, Zorlu F: A case of iatrogenic ureter injury: Recent diagnostic and treatment. $J$ Urolog Surg 3:95-97, 2016

7. Ferrara M, Kann BR: Urological injuries during colorectal surgery. Clin Colon Rectal Surg 32(3):196-203, 2019
8. Garg N, Panwar P, Devana SK, Mohan SMR, Mandal AK: Ureteric injury after lumbosacral microdiscectomy: A case report and review of literature. Urol Ann 9(2):200-203, 2017

9. Krantz TE, Mcferren SC, Riley JM, Dunivan GC, Alba FM: "Show Me How" video tips and tricks for performing a retrograde pyelogram. Urology 129:234, 2019

10. Kuang L, Chen Y, Li L, Lü G, Wang B: Applying the mini-open anterolateral lumbar interbody fusion with self-anchored stand-alone polyetheretherketone cage in lumbar revision surgery. Biomed Res Int 2016:1758352, 2016

11. Kusano S, Dezawa A, Yoshihara K, Katoh K: Anterolateral Approach to the lumbar spine. In: Dezawa A, Chen PQ, Chung JY (eds), State of the Art for Minimally Invasive Spine Surgery, Tokyo: Springer, 2005:107-116

12. Levitske C, Gollapudi K, Pak S, Liang L: Urology case reports repair of iatrogenic ureteral injury secondary to breast cancer metastasis. Urol Case Rep 28:101024, 2020

13. Mehren C, Mayer HM, Zandanell C, Siepe CJ, Korge A: The oblique anterolateral approach to the lumbar spine provides access to the lumbar spine with few early complications. Clin Orthop Relat Res 474(9):2020-2027, 2016

14. Sokolis DP: In vitro study of age-related changes in human ureteral failure properties according to region, direction, and layer. J Engineering in Medicine 233(5):570-583, 2019

15. Wagner WH, Regan JJ, Leary SP, Lanman TH, Johnson JP, Rao RK, Cossman DV: Access strategies for revision or explantation of the Charité lumbar artificial disc replacement. J Vasc Surg 44(6):1266-1272, 2006 